\title{
Avaliação ultra-sonográfica da genitália interna de meninas com puberdade precoce central idiopática antes e durante o tratamento com análogo de GnRH
}

\author{
Ultrasound evaluation of internal genitalia of girls with idiopathic central precocious \\ puberty before and during treatment with GnRH analogs
}

Ângela Clotilde Ribeiro Falanga e Lima' ${ }^{1}$, Renan Magalhães Montenegro Júnior ${ }^{2}$, Francisco Edson de Lucena Feitosa ${ }^{3}$, Rosa Maria Salani Mota ${ }^{4}$, Carlos Augusto Alencar Júnior ${ }^{5}$, Zenilda Vieira Bruno ${ }^{6}$

RESUMO

Objetivo: verificar, com o emprego da ultra-sonografia pélvica, a existência de mudanças na genitália interna de meninas com puberdade precoce central submetidas a tratamento com análogo do hormônio liberador de gonadotrofinas $(\mathrm{GnRH})$. Métodos: a ultra-sonografia pélvica foi realizada em 18 meninas com diagnóstico de puberdade precoce central idiopática, antes e três meses após o inicio do tratamento com análogo de $\mathrm{GnRH}$, para avaliar o impacto da terapia na genitália interna feminina. Foram avaliados os volumes uterino e ovariano, o diâmetro longitudinal do útero, a relação entre os diâmetros longitudinais do corpo e colo uterinos, a relação entre os diâmetros ântero-posteriores do corpo e colo uterinos e o eco endometrial. Para a análise estatística foi aplicado o teste de Shapiro-Willks para verificação da normalidade dos dados. Para os dados em que a normalidade foi satisfeita, foi aplicado o teste $t$ de Student. Para os dados cuja distribuição não foi normal aplicou-se o teste não paramétrico (teste do sinal).Resultados: após o tratamento houve redução estatisticamente significante da média dos volumes uterino (de 5,4 para $3,0 \mathrm{~cm}^{3}, \mathrm{p}<0,001$ ) e ovariano (de 2,2 para $1,1 \mathrm{~cm}^{3}, \mathrm{p}=0,004$ ), da média do diâmetro longitudinal do útero (de 4,2 para $3,4 \mathrm{~cm}, \mathrm{p}=0,001$ ) e da média do eco endometrial (de 1,8 para $0,6 \mathrm{~mm}, \mathrm{p}=0,018$ ).Conclusão: em meninas com puberdade precoce a ultra-sonografia pélvica é útil para avaliar a eficácia do tratamento com análogo de GnRH. Os principais parâmetros de resposta à terapia são as diminuições dos volumes uterino e ovariano, a redução do diâmetro longitudinal do útero e a atrofia ou ausência do eco endometrial.

PALAVRAS-CHAVE: Puberdade precoce/terapia; Pelve/ultrasonografia; Útero/ultrasonografia; Gonadorrelina/uso terapêutico; Estudos de coortes

\section{ABSTRACT}

Purpose: to verify, through pelvic ultrasound, the existence of changes in the internal genitalia of girls with central precocious puberty, submitted to treatment with gonadotrophin-releasing hormone (GnRH) analogs. Methods: pelvic ultrasound was performed in 18 girls with idiopathic central precocious puberty, before and after three months of onset of the treatment with $\mathrm{GnRH}$ analogs, to investigate the impact of the therapy on the internal genitalia. Ovarian and uterine volumes, uterine longitudinal length, relation between the longitudinal diameter of the uterine corpus and the uterine cervix, the relation between the anteriorposterior diameter of the uterine corpus and the uterine cervix, and endometrial echogenicity were evaluated. Statistical analysis was performed through Shapiro-Willkis's test, to assess data normality. When normality was present, Student's test $t$ was applied. For data without normality, a non-parametric test (the signal test) was used. Results: after therapy, statistically significant decline of the mean uterine volume (from $5.4 \mathrm{~cm}^{3}$ to $3.0 \mathrm{~cm}^{3}, \mathrm{p}<0.001$ ), of the mean ovarian volume (from $2.2 \mathrm{~cm}^{3}$ to $1.1 \mathrm{~cm}^{3}, \mathrm{p}=0.004$ ), of the mean uterine longitudinal length (from $4.2 \mathrm{~cm}$ to $3.4 \mathrm{~cm}, \mathrm{p}=0.001$ ), and of the mean endometrial echogenicity (from $1.8 \mathrm{~mm}$ to $0.6 \mathrm{~mm}, \mathrm{p}=0.018$ ) occurred. Conclusion: In girls with idiopathic central precocious puberty, pelvic ultrasound is a valid method to assess the efficacy of treatment with GnRH analogs. The main parameters of the therapeutic response were the decrease of uterine and ovarian volume, of uterine longitudinal length, and atrophy or absence of endometrial echogenicity.

KEYWORDS: Puberty, precocious/therapy; Pelvis/ultrasonography; Uterus/ultrasonography; Gonadorelin/therapeutic use; Cohort studies

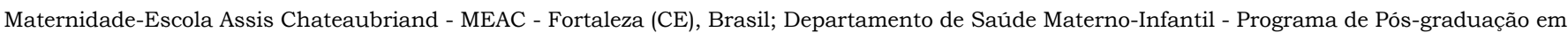
Tocoginecologia da Universidade Federal do Ceará - UFC - Fortaleza (CE), Brasil.

1 Médica Ginecologista e Mestra em Tocoginecologia pela Universidade Federal do Ceará - UFC - Fortaleza (CE), Brasil.

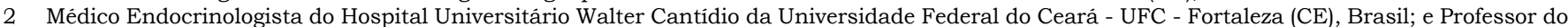
Curso de Mestrado de Educação em Saúde da Universidade de Fortaleza - UNIFOR - Fortaleza (CE), Brasil.

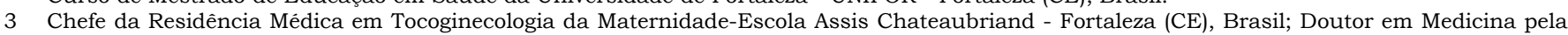
Universidade Estadual de Campinas - UNICAMP - Campinas (SP), Brasil.

4 Professora Adjunta IV do Departamento de Estatística e Matemática Aplicada da UFC.

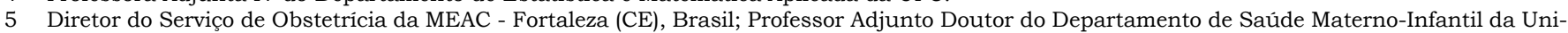
versidade Federal do Ceará - UFC - Fortaleza (CE), Brasil.

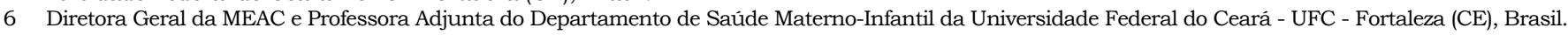
Correspondência: Zenilda Vieira Bruno

Rua Paula Ney, 55 - Apto 901 - Aldeota - 60140-200 - Fortaleza - CE - Fone: (85) 3264-5768 - e-mail: zenildabruno@terra.com.br 


\section{Introdução}

A puberdade é a fase do desenvolvimento humano que compreende o período de transição entre a infância e a vida adulta, na qual ocorre uma seqüência de modificações neurológicas, hormonais e físicas que resultam na maturação sexual, permitindo ao organismo atingir sua forma e capacidade funcional de adulto capaz para a reprodução. Baseados em dados epidemiológicos considera-se como precoce o aparecimento dos caracteres sexuais secundários antes dos oito anos em meninas, idade abaixo de dois desvios padrão da média de seu início normal ${ }^{1,2}$. A incidência da precocidade sexual é estimada em um em 5.000 a 10.000 casos, com 2000 casos novos diagnosticados por ano $\mathrm{an}^{3,4}$. Ocorre predominantemente em meninas, sendo citada relação entre os sexos feminino:masculino desde $3: 1$ até 23:14,5. Em estudo epidemiológico realizado na Dinamarca, entre 1993 e 2001, evidenciou-se que $0,2 \%$ das meninas desenvolveram, no período, alguma forma de precocidade sexual ${ }^{6}$.

A puberdade precoce pode ser classificada em incompleta, periférica, pseudopuberdade ou independente do hormônio liberador das gonadotrofinas (GnRH), conseqüente à produção hormonal ovariana ou adrenal ou à exposição aos esteróides sexuais. Pode, por outro lado, ser completa, central, verdadeira ou dependente do $\mathrm{GnRH}$, conseqüente a uma reativação prematura do eixo hipotálamohipófise-gonadas, semelhante à que existe na puberdade normal. Em meninas, a puberdade precoce central é, em 70 a $90 \%$ dos casos, de causa idiopática ${ }^{7}$. Elevação significante do LH (basal ou após estímulo) caracteriza a puberdade como de origem central ou GnRH dependente, significando que o eixo hipotálamo-hipófise-gônadas está ativado.

O tratamento da puberdade precoce de origem central está indicado para prevenir a progressão da puberdade, o inoportuno crescimento da estatura, os possiveis distúrbios psicossociais que a criança pode apresentar e a baixa estatura ${ }^{8}$. Na terapia, há mais de vinte anos, são utilizados os análogos de GnRH. Os efeitos físicos da supressão não são limitados ao desenvolvimento ósseo. $\mathrm{Na}$ maioria das meninas não há aumento das mamas e ocorre redução dos tamanhos do útero e ovários $^{9}$. Destaque-se, ainda, que a terapia melhora a estatura final das crianças e não oferece efeitos adversos sobre a função reprodutiva após a suspensão da medicação ${ }^{10}$.

A ultra-sonografia pélvica provou ser técnica não invasiva e precisa na avaliação da genitália interna de meninas com puberdade precoce central. Vários parâmetros ultra-sonográficos são citados como indicadores do efeito da secreção estrogênica, destacando-se o aumento dos volumes uterino ${ }^{11-14}$ e ovariano ${ }^{15-17}$, o incremento do comprimento longitudinal do útero ${ }^{16}$ e da relação entre as medidas do corpo e do colo uterinos ${ }^{17,18}$ e a presença do eco endometrial ${ }^{11,14,17,18}$. Após e durante o tratamento, a ultra-sonografia pélvica pode monitorizar se essas mudanças puberais precocemente desenvolvidas estão regredindo.

Nosso trabalho visa avaliar se ocorrem mudanças na genitália interna de meninas com puberdade precoce central idiopática submetidas a tratamento com análogos de GnRH por meio da ultra-sonografia pélvica.

\section{Métodos}

Estudo de coorte prospectivo e descritivo das mudanças ultra-sonográficas evidenciadas nos órgãos genitais internos de crianças do sexo feminino com diagnóstico de puberdade precoce central idiopática, submetidas a tratamento com análogo de GnRH. Foram realizados exames ultra-sonográficos pélvicos, sendo um antes e outro três meses após o início da terapia supressiva. Os casos foram incluídos no período de maio de 2003 a junho de 2004, no Serviço de Ultra-sonografia da Maternidade-Escola Assis Chateaubriand, da Universidade Federal do Ceará.

Foram incluídas 18 meninas com diagnóstico de puberdade precoce verdadeira idiopática com indicação para tratamento clínico com análogo de GnRH, atendidas no Ambulatório de Endocrinologia do Hospital Universitário Walter Cantídio, da Faculdade de Medicina da Universidade Federal do Ceará, e no Ambulatório de Puberdade Precoce, do Instituto de Diabetes e Hipertensão do Governo do Estado do Ceará. O diagnóstico da puberdade precoce verdadeira idiopática foi efetuado nos serviços de origem. Os resultados dos exames ultra-sonográficos inicial e após os três meses de terapia foram informados aos responsáveis pelo acompanhamento das pacientes.

Foram excluídas crianças com puberdade precoce secundária a outras etiologias de precocidade sexual, as que haviam feito uso prévio dos análogos de GnRH ou de outras formas de tratamento e as que usaram a terapia supressora de forma irregular. O estudo foi aprovado pelo Comitê de Ética em Pesquisa do Complexo Hospitalar da Universidade Federal do Ceará.

O exame foi realizado e interpretado sempre pelo autor da pesquisa. Todos os exames foram efetuados no mesmo aparelho, modelo Diasonics Multisync M 500, da General Electrics, com trans- 
dutores convexos de 3,5 $\mathrm{mHz}$. O método empregado foi o convencional, com a paciente em decúbito dorsal, bexiga repleta, obtida após a administração oral de fluidos e retenção urinária voluntária. Mensuraram-se os diâmetros longitudinal, transverso e ântero-posterior do útero e ovários, quando visualizados. Os diâmetros longitudinais do corpo e do colo uterinos foram também mensurados, sendo calculada a relação entre eles. Da mesma forma, mensuraram-se os diâmetros ântero-posteriores do corpo e do colo uterinos sendo também calculada a relação entre as medidas efetuadas. Foi medido o eco endometrial, em milímetros, em corte sagital do útero, após ampliação da imagem até permitir a mensuração em casas decimais. Todas as medidas foram efetuadas três vezes, sendo o resultado final a média das mensurações. As variáveis ultra-sonográficas estudadas, no início e no transcorrer do tratamento, foram os volumes uterino e ovariano, a espessura do eco endometrial, o diâmetro longitudinal do útero, a relação entre os diâmetros longitudinais do corpo e colo uterinos e a relação entre os diâmetros ântero-posteriores do corpo e colo uterinos.

Os volumes uterino e ovariano foram calculados pela fórmula da elipse (volume $=$ diâmetro longitudinal $\mathrm{x}$ diâmetro ântero-posterior $\mathrm{x}$ diâmetro transverso x 0,5233). Quando apenas um ovário foi visualizado utilizou-se o volume deste ovário para fins de cálculo. Quando os dois ovários foram mensurados empregou-se a média dos volumes obtidos para análise. Foram utilizados, como padrão de normalidade para as diferentes faixas etárias, os valores ovarianos e uterinos previamente definidos na literatura ${ }^{19}$.

Empregou-se o acetato de leuprolida, por via intramuscular, nas doses de 7,5 mg. A primeira dose foi aplicada após a realização da ultra-sonografia inicial da pesquisa, a segunda dose 15 dias após a primeira e, a partir daí, doses subseqüentes a cada 21 dias. A suspensão do tratamento ficou a cargo dos serviços ambulatoriais que acompanharam as pacientes. Todas as meninas fizeram uso de cinco doses do análogo antes da reavaliação ultra-sonográfica.

Para a análise estatística foi aplicado o teste de Shapiro-Willks para verificação da normalidade dos dados. Nos casos em que não se verificou normalidade, foi feita a transformação da variável por meio do logaritmo neperiano (ln) para a verificação da possivel normalidade da distribuição residual. Para os dados em que a normalidade foi satisfeita, e para aqueles cuja transformação logaritmica satisfez a normalidade, foi aplicado o teste $t$ de Student para populações pareadas. Para os dados cuja distribuição não foi normal aplicou- se o teste não paramétrico (teste do sinal). O nível descritivo dos testes foi para teste unilateral. Os resultados foram expressos em média \pm desvio padrão, sendo adotado o nivel de significância de $5 \%$, onde $\mathrm{p} \leq 0,05$.

\section{Resultados}

Todas as participantes do estudo foram avaliadas com ultra-sonografia pélvica imediatamente antes de iniciar o tratamento com análogo do GnRH e após o período de três meses de tratamento. Em todos os casos foi possivel avaliar o útero e pelo menos um dos ovários. Em apenas uma paciente não foi possivel identificar os dois ovários. Não foi possivel mensurar o eco endometrial em três meninas, tanto antes como após o tratamento. Nas outras 15 crianças o eco endometrial foi mensurado antes da terapia. Após o tratamento não foi possivel medir o endométrio em sete meninas.

O volume uterino foi, em média, de $5,4 \mathrm{~cm}^{3}$ $( \pm 5,2)$ e de $3,0 \mathrm{~cm}^{3}( \pm 2,6)$, com mediana de 3,6 e de $2,1 \mathrm{~cm}^{3}$, antes e após o uso do análogo, respectivamente. Observa-se que a média do volume uterino diferiu significativamente $(\mathrm{p}<0,001)$, quando se comparou o volume antes e depois de efetuado o tratamento com análogos (Tabela 1).

A medida do diâmetro longitudinal do útero foi, em média, de $4,2 \mathrm{~cm}( \pm 1,0)$ e de $3,4 \mathrm{~cm}( \pm 0,7)$, com mediana de 4,0 e de $3,3 \mathrm{~cm}$, antes e após o uso do análogo, respectivamente. Observa-se que a média do diâmetro longitudinal do útero diferiu significativamente $(\mathrm{p}=0,001)$, antes e depois de efetuado o tratamento (Tabela 1).

A relação entre o diâmetro longitudinal do corpo uterino e o diâmetro longitudinal do colo foi, em média, de $1,9( \pm 0,4)$ e de $1,7( \pm 0,3)$, com medianas de 1,9 e de 1,6, antes e após o uso do análogo, respectivamente. As diferenças entre estes resultados não foram significantes $(p=0,061$; (Tabela 1).

A relação entre o diâmetro ântero-posterior do corpo uterino e o diâmetro ântero-posterior do colo foi, em média, de $1,3( \pm 0,2)$ e de $1,2( \pm 0,2)$, com medianas de 1,3 e de 1,3, antes e após o uso do análogo, respectivamente. As diferenças também não foram estatisticamente significantes $(\mathrm{p}=0,358$; Tabela 1$)$.

A espessura do eco endometrial apresentou médias de $1,8 \mathrm{~mm}( \pm 1,5)$ e $0,6 \mathrm{~mm}( \pm 0,6)$, com medianas de 1,3 e $0,8 \mathrm{~mm}$, antes e depois do uso do análogo, respectivamente. Houve diferença significante $(p=0,018)$ entre as médias da espessura do eco endometrial previamente e posteriormente ao tratamento (Tabela 1 ). 
Quanto ao volume ovariano as médias foram de $2,2 \mathrm{~cm}^{3}( \pm 1,7)$ e de $1,1 \mathrm{~cm}^{3}( \pm 0,7)$, com medianas de 1,7 e $1,0 \mathrm{~cm}^{3}$ (antes e depois do tratamento, respectivamente). Observa-se que, em média, o volume ovariano diferiu significativamente $(p=0,004)$ quando comparado seu valor prévio à terapia em relação ao observado após o tratamento (Tabela 1).

\section{Discussão}

A ultra-sonografia é importante instrumento na avaliação da pelve infantil. A ausência de efeitos deletérios sobre o organismo e, especialmente, a capacidade para diferenciar tecidos sólidos e líquidos e obter imagens em múltiplos planos fizeram do método o ideal para avaliar a pelve feminina ${ }^{20}$. Na criança com puberdade precoce é usada principalmente para determinar a morfologia da anatomia da genitália interna (volume das gônadas, forma e tamanho uterinos, avaliação do eco endometrial) e também excluir a presença de neoplasias ovarianas.

Os ovários da menina pré-púbere possuem aproximadamente $0,5 \mathrm{~cm}^{3}$ de volume, aumentando gradualmente em resposta à ação das gonadotrofinas. Volume maior ou igual a $1 \mathrm{~cm}^{3}$ indica ação gonadotrófica e, conseqüentemente, início da puberdade, época em que atingirá $4 \mathrm{~cm}^{3}$ rapidamente ${ }^{20}$.

O útero é o órgão do aparelho reprodutor feminino que apresenta crescimento mais rápido durante a puberdade. Seu volume aumentará de $1 \mathrm{~cm}^{3}$, aos sete anos de idade, até cerca de $25 \mathrm{~cm}^{3}$, na época da menarca. O corpo uterino, que na in- fância é menor do que o colo, cresce mais, ficando com volume semelhante ao do colo próximo da menarca. No fim da puberdade, atingirá tamanho três vezes maior do que o colo ${ }^{21}$.

Vários achados ultra-sonográficos são citados como indicadores da existência da puberdade precoce. Em 151 meninas com diversas desordens do desenvolvimento puberal, em comparação com 131 crianças sadias, na mesma faixa etária, as médias dos volumes uterino e ovariano foram significativamente maiores nas portadoras da alteração ${ }^{12}$. Resultados idênticos foram evidenciados por outros autores ${ }^{17,22}$.

A presença do eco endometrial e o comprimento uterino maior do que dois desvios padrões para a média da idade específica tiveram especificidades de 98 e 95\% e sensibilidades de 42 e 46\%, respectivamente, para o diagnóstico da puberdade precoce $^{7}$. Em outra pesquisa, a avaliação do volume uterino e a presença do eco endometrial também apresentaram boas especificidades, de 90 e $80 \%$, respectivamente, e sensibilidades de 78 e $56 \%$, respectivamente $^{11}$. O aumento da relação corpo-colo também foi referido como um dos bons indicadores do incremento da secreção estrogênica ${ }^{18}$.

A ultra-sonografia também pode ser útil no diagnóstico diferencial das várias formas de precocidade sexual. Entre 69 crianças com desenvolvimento mamário e/ou púbico antes dos oito anos de idade, evidenciou-se que, nas com puberdade precoce verdadeira, a presença do eco endometrial e o aumento do volume uterino eram mais freqüentes que naquelas portadoras de telarca ou pubarca isoladas ${ }^{14}$.

Tabela 1 - Variáveis ultra-sonográficas avaliadas antes e durante o tratamento com análogo de GnRH.

\begin{tabular}{|c|c|c|c|c|c|c|c|}
\hline Variável & $\mathrm{n}$ & Média & DP & Mínimo & Mediana & Máximo & $\mathrm{p}^{\#}$ \\
\hline $\operatorname{VU}\left(\mathrm{cm}^{3}\right)^{*}$ & 18 & 5,4 & 5,2 & 1,6 & 3,6 & 23,5 & \\
\hline ** & 18 & 3,0 & 2,6 & 0,9 & 2,1 & 12,7 & $<0,001^{(1)}$ \\
\hline $\operatorname{DLU}(\mathrm{cm})^{*}$ & 18 & 4,2 & 1,0 & 2,5 & 4,0 & 6,8 & \\
\hline ** & 18 & 3,4 & 0,7 & 2,2 & 3,3 & 4,9 & $0,001^{(1)}$ \\
\hline DL corpo/colo * & 18 & 1,9 & 0,4 & 1,3 & 1,9 & 2,9 & \\
\hline ** & 18 & 1,7 & 0,3 & 1,2 & 1,6 & 2,5 & $0,061^{(1)}$ \\
\hline DAP corpo/colo * & 18 & 1,3 & 0,2 & 0,7 & 1,3 & 1,8 & \\
\hline ** & 18 & 1,2 & 0,2 & 0,8 & 1,3 & 1,7 & $0,358^{(1)}$ \\
\hline Eco end. $(\mathrm{mm})^{*}$ & 15 & 1,8 & 1,5 & 1,1 & 1,3 & 7,3 & \\
\hline ** & 15 & 0,6 & 0,6 & 0,0 & 0,8 & 1,8 & $0,018^{(2)}$ \\
\hline VO $\left(\mathrm{cm}^{3}\right)^{*}$ & 18 & 2,2 & 1,7 & 0,3 & 1,7 & 7,2 & \\
\hline$* *$ & 18 & 1,1 & 0,7 & 0,3 & 1,0 & 3,5 & $0,004^{(1)}$ \\
\hline
\end{tabular}

$\mathrm{DP}=$ desvio padrão.

DAP corpo/colo: relação entre os diâmetros antero-posteriores do corpo e colo; DL corpo/colo: relação entre os diâmetros longitudinais do corpo e colo; DLU: diâmetro longitudinal do útero; eco end.: eco endometrial; VO: volume ovariano; VU: volume uterino.

*Antes de efetuado o tratamento com análogo.

**Durante o tratamento com análogo.

\#p com valor descritivo de teste unilateral.

(1): Teste $t$ de Student.

(2): Teste do sinal. 
O aumento dos volumes do útero e/ou ovários associado às mudanças da morfologia uterina e à presença do eco endometrial podem, portanto, ser considerados sinais ultra-sonográficos da puberdade precoce ${ }^{15}$.

Em virtude destes achados a ultra-sonografia pélvica tem, de fato, se tornado instrumento de rotina na avaliação inicial de meninas que irão se submeter à terapia supressora. Além disso, passou a ser utilizada para monitorar a reversão das alterações decorrentes da precocidade puberal.

A avaliação da anatomia dos órgãos pélvicos pode ser dificultada pela abordagem transabdominal por vários motivos, como obesidade, necessidade da bexiga repleta, alças intestinais dilatadas, que podem ser superados pela via transvaginal. Embora nossas pacientes estejam impedidas de realizar a ultra-sonografia transvaginal, pela própria idade e integridade do hímen, pela via transabdominal, no exame inicial, visualizou-se o útero em todos os casos, os dois ovários em $94 \%$ dos exames e pelo menos um ovário na totalidade das avaliações. No primeiro exame o eco endometrial foi mensurado em $83,3 \%$ das pacientes.

Em nosso trabalho avaliamos o uso da ultrasonografia na monitorização da eficácia supressiva da terapia com análogo de GnRH em meninas com diagnóstico de puberdade precoce central idiopática, por meio da observação das mudanças morfológicas do útero e ovários. Observamos que tanto o volume uterino quanto o diâmetro longitudinal do útero tiveram diminuição de seus valores durante a terapia supressiva com análogo de GnRH. Em relação à espessura do eco endometrial, também se observou diferença significante. Encontramos aumento do volume ovariano em todas as pacientes com diagnóstico de puberdade precoce central. Após o tratamento, houve diminuição significativa do seu volume em relação aos valores prévios ao tratamento.

A diminuição do volume ovariano e do comprimento do útero, mesmo com valores ainda acima dos limiares da normalidade, já pode indicar início da supressão do eixo ${ }^{16}$. Em 33 meninas com puberdade precoce central idiopática havia aumento do útero e ovários na maioria das pacientes antes do tratamento. Depois de três meses de terapia, os volumes uterino e ovariano diminuíram significativamente para valores próprios para a idade ${ }^{23}$. Os volumes uterino e ovariano deverão retornar aos limites normais entre três e doze meses após o início do tratamento ${ }^{24}$. Espera-se que, após um ano de terapia, os volumes estejam normais na maioria das pacientes ${ }^{23}$.

Nossos resultados reafirmam o valor da ultra-sonografia como instrumento fidedigno na investigação da genitália interna feminina em pacientes com precocidade sexual. A significante redução dos volumes uterino e ovariano, a diminuição do comprimento longitudinal do útero e a supressão do eco endometrial durante o tratamento refletem sua utilidade na monitorização do sucesso da terapia com análogo de GnRH em meninas com puberdade precoce central.

\section{Referências}

1. Antoniazzi F, Zamboni G. Central precocious puberty: current treatment options. Paediatr Drugs. 2004;6(4):211-31.

2. Aguiar AL, Couto-Silva AC, Freitas I, Cruz T, Rodrigues M, Adan LF. Puberdade precoce central idiopática em meninas no estado da Bahia. Arq Bras Endocrinol Metab. 2005;49(4): 536-41.

3. Diaz A, Danon M. Recent advances in the diagnosis and treatment of precocious puberty. Indian $\mathrm{J}$ Pediatr. 2000;67(3):211-5.

4. Kappy MS, Ganong CS. Advances in the treatment of precocious puberty. Adv Pediatr. 1994;41:223-61.

5. Bridges NA, Christopher JA, Hindmarsh PC, Brook CG. Sexual precocity: sex incidence and aetiology. Arch Dis Child. 1994;70(2):116-8.

6. Teilmann G, Pedersen CB, Jensen TK, Skakkebaek $\mathrm{NE}$, Juul A. Prevalence and incidence of precocious pubertal development in Denmark: an epidemiologic study based on national registries. Pediatrics. 2005;116(6):1323-8.

7. Cisternino M, Arrigo T, Pasquino AM, Tinelli C, Antoniazzi F, Beduschi L, et al. Etiology and age incidence of precocious puberty in girls: a multicentric study. J Pediatr Endocrinol Metab. 2000;13 Suppl 1: 695-701.

8. Lee PA. Central precocious puberty: an overview of diagnosis, treatment, and outcome. Endocrinol Metab Clin North Am. 1999;28(4):901-18.

9. García Bruce H, Youlton RR, Burrows Argote R, Catanni Ortega A. Consenso sobre el diagnóstico y tratamiento de la pubertad precoz central. Rev Med Chile. 2003;131(1):95-110.

10.Tanaka T, Niimi H, Matsuo N, Fujieda K, Tachibana K, Ohyama K, et al. Results of long-term follow-up after treatment of central precocious puberty with leuprorelin acetate: evaluation of effectiveness of treatment and recovery of gonadal function. The TAP-144-SR Japanese study group on central precocious puberty. $\mathrm{J}$ Clin Endocrinol Metab. 2005;90(3):1371-6.

11.Battaglia C, Regnani G, Mancini F, Iughetti L, Venturoli S, Flamigni C. Pelvic sonography and uterine artery color Doppler analysis in the diagnosis of female precocious puberty. Ultrasound Obstet Gynecol. 2002;19(4):386-91. 
12.Salardi S, Orsini LF, Cacciari E, Partesotti S, Brondelli L, Cicognani A, et al. Pediatric ultrasonography in girls with precocious puberty, congenital adrenal hyperplasia, obesity or hirsutism. J Pediatr. 1988; $112(6): 880-7$.

13.Haber HP, Wollmann HA, Ranke MB. Pelvic ultrasonography: early differentiation between isolated premature thelarche and central precocious puberty. Eur J Pediatr. 1995;154(3):182-6.

14.Battaglia C, Mancini F, Regnani G, Persico N, Iughetti L, De Alysio D. Pelvic ultrasound and color Doppler findings in different isosexual precocities. Ultrasound Obstet Gynecol. 2003;22(3):277-83.

15. Teixeira RJ, Silva VCG, Freitas JR, Henriques JLS, Guimarães MM. Ultra-sonografia pélvica em 140 meninas normais pré e pós-puberais. Arq Bras Endocrinol Metab. 1999;43(3):210-6.

16.Ambrosino MM, Hernanz-Schulman M, Genieser NB, Sklar CA, Fefferman NR, David R. Monitoring of girls undergoing medical therapy for isosexual precocious puberty. J Ultrasound Med. 1994;13(7):501-8.

17.Griffin IJ, Cole TJ, Duncan KA, Hollman AS, Donaldson MD. Pelvic ultrasound findings in different forms of sexual precocity. Acta Paediatr. 1995;84(5): 544-9.

18.Cassio A, Cacciari E, Zucchini S, Balsamo A, Diegoli
M, Orsini F. Central precocious puberty: clinical and imaging aspects. J Pediatr Endocrinol Metab. 2000;13 Suppl 1:703-8.

19. Orsini LF, Salardi S, Pilu G, Bovicelli L, Cacciari E. Pelvic organs in premenarcheal girls: real-time ultrasonography. Radiology. 1984;153(1):113-6.

20.Ziereisen F, Guissard G, Damry N, Avui EF. Sonographic imaging of the paediatric female pelvis. Eur Radiol. 2005;15(7):1296-309.

21.Bridges NA, Cooke A, Healy MJ, Hindmarsh PC, Brook CG. Growth of the uterus. Arch Dis Child. 1996;75(4):330-1.

22.Buzi F, Pilotta A, Dordoni D, Lombardi A, Zaglio S, Adlard P. Pelvic ultrasonography in normal girls and in girls with pubertal precocity. Acta Paediatr. 1998;87(11):1138-45.

23.Jensen AM, Brocks V, Holm K, Laursen EM, Müller J. Central precocious puberty in girls: internal genitalia before, during and after treatment with long-acting gonadotropin-releasing hormone analogues. J Pediatr. 1998;132(1):105-8.

24. Carel JC, Lahlou N, Guazzarotti L, Joubert-Collin M, Roger M, Colle M, et al. Treatment of central precocious puberty with depot leuprorelin. French Leuprorelin Trial Group. Eur J Endrocrinol 1995;132(6): 699-704. 